\title{
Plunging into the pool of death: Imagining a dangerous outcome influences distance perception
}

Jeanine K. . Stefanucci

Kyle T. Gagnon

Christopher L. Tompkins

William \& Mary

Kendall E. Bullock

William \& Mary

\section{Recommended Citation}

Stefanucci, J. K., Gagnon, K. T., Tompkins, C. L., \& Bullock, K. E. (2012). Plunging into the pool of death: Imagining a dangerous outcome influences distance perception. Perception, 41(1), 1-11. 


\title{
Plunging into the pool of death: Imagining a dangerous outcome influences distance perception
}

\author{
Jeanine K Stefanucci, Kyle T Gagnon, Christopher L Tompkins§, Kendall E Bullock§ \\ Department of Psychology, University of Utah, 380 S 1530 E, Salt Lake City, UT 84112, USA; \\ e-mail: jeanine.stefanucci@psych.utah.edu; § Department of Psychology, College of William \& Mary, \\ Williamsburg, VA 23187, USA \\ Received 29 September 2011, in revised form 21 January 2012
}

\begin{abstract}
We examined whether manipulating the imagined consequences of falling would influence the perception of height, distance, and size. In experiment 1 , height and size perception were measured when participants stood at a short height $(0.89 \mathrm{~m})$ or a medium height $(1.91 \mathrm{~m})$ above either an empty pool or a pool filled with a bed of nails. Participants who viewed the bed of nails and imagined falling into it estimated both the height as taller and the size of the bed of nails as larger than participants who imagined falling into an empty pool. In a second experiment, participants overestimated the horizontal ground distance to and across the bed of nails after being told to imagine jumping over it. Overall, these experiments suggest that costs associated with imagined actions can influence the perception of both vertical and horizontal extents that are not inherently dangerous.
\end{abstract}

\section{Introduction}

Though traditional approaches to the study of visual perception argue that vision is an encapsulated process seemingly unaffected by non-visual factors like emotion (Pylyshyn 2003), recent studies on the perception of spatial layout call this claim into question. For example, Stefanucci and Proffitt (2009) showed that the perception of height is related to whether or not the observer is afraid of heights. Similarly, Teachman et al (2008) found that a selected sample of people high in height fear exhibited more bias in their perception of height when compared to those low in height fear. These preliminary findings provide support for the argument that non-optical variables, including physiological potential, intention, and emotion, can influence visual perception (cf Proffitt 2006). However, the findings from these prior studies cannot determine causal directions for the relationship between emotion and perception, given that fear was not directly manipulated. Furthermore, all of the previous studies examined the influence of fear on perception in situations in which the environment was inherently dangerous, whereas these studies will address whether fear can influence non-dangerous environmental perception.

Research on height perception demonstrates that individuals overestimate vertical distances at a greater rate from the top than from the bottom (Jackson and Cormack 2007; Stefanucci and Proffitt 2009), and fear of heights is correlated with this overestimation (Stefanucci and Proffitt 2009). Those with a greater fear of heights estimate the height as taller, especially from the top, and this relationship is pronounced when examining groups who have extreme fears (Teachman et al 2008). Furthermore, these perceptual distortions are independent of explicit and implicit cognitive associations involved in height fear. Other recent research has also found that participants high in height fear are particularly vulnerable to manipulations in which they are asked to imagine falling from a height in order to increase the perceived costs of acting on the height (Clerkin et al 2009). Though participants both high and low in height fear overestimated a high height more when they imagined falling from it, imagining falling increased height estimates for the high-fear group more than the low-fear participants. 
The results show that enhancing awareness of the consequences of falling can increase height estimates in clinical samples.

The aforementioned experiments support the hypothesis that fear can influence perception, specifically that of slant and height when the consequences for falling may be greater. These findings suggest that dangerous extents or environments, in general, could be perceived differently. In order to determine whether this claim is true, three potential hypotheses should be tested to rule out alternative explanations. First, we will test non-dangerous heights to determine whether or not they elicit fear and, if they do not elicit as much fear, then we would expect perceptions not to change or to change very little. The previous studies have only examined the relationship between fear and height perception in heights over $6 \mathrm{~m}$. We assume that people will not view a $2 \mathrm{~m}$ height as dangerous, but this is an open question. If they do not believe it to be dangerous, then this will allow us to test whether directly manipulating the consequences of falling can alter height perception across a range of heights that are not perceived to be dangerously high. Finally, our work will test whether altering the dangerous consequences for action can affect another parameter of space perception that is definitely not inherently dangerous: the perception of horizontal distances in a hallway. This question is especially important to answer, because it will elucidate whether the previously observed effects are specific to dangerous slants and heights or, alternatively, if fear can influence perceptions of spatial dimensions that are relevant to the source of the fear but inherently non-dangerous.

In the first experiment, participants were asked to estimate shorter, non-dangerous heights. Particularly in question was whether participants (i) perceived the heights as dangerous and (ii) if those who were asked to imagine falling into a bed of nails would perceive the height as taller and the pool as larger than participants who imagined falling with no additional consequences at the base of the height. In the second experiment, the perception of a horizontal distance was examined by asking participants to imagine jumping over the pool of nails. In this manner, less dangerous parameters of environmental layout, including horizontal ground distance and shorter heights, could be assessed to test for an influence of consequences for action.

\section{Experiment 1}

In this experiment, the consequences of falling from a short height $(0.89 \mathrm{~m})$ and a medium height $(1.91 \mathrm{~m})$ were manipulated by having participants imagine falling into a pool containing a bed of nails or an empty pool. We hypothesized that when a dangerous environment was present at the base of the height, thereby increasing the consequences for falling, participants would overestimate the heights when compared to participants not presented with consequences for falling. We also examined whether these heights were perceived as dangerous or not given the consequences of falling were less severe.

\subsection{Method}

2.1.1 Participants. Forty-seven (twenty-eight female, nineteen male) undergraduate students (mean age $=19$ years, $\mathrm{SD}=1.09$ years) participated in the study to fulfill a course requirement. For one participant, both the imagery and injury ratings were missing due to experimenter error.

2.1.2 Stimuli and apparatus. The apparati used in this experiment included two inflatable swimming pools that were $1.17 \mathrm{~m}$ in diameter. In the control condition the pool was left empty, and in the experimental condition the pool contained a nail bed in the bottom. The nail bed was a circular piece of $1.9 \mathrm{~cm}$ thick plywood that was $1.14 \mathrm{~m}$ in diameter and fit tightly in the pool. Nails of varying thickness $(0.32-1.27 \mathrm{~cm}$ diameter $)$ and length $(7.62-15.24 \mathrm{~cm})$ were nailed through the plywood to stick up vertically. 
The locations chosen for testing were the $0.89 \mathrm{~m}$ loading dock of an academic building and a $1.91 \mathrm{~m}$ height (atop a brick wall) just outside of the campus library. The pools were placed at the bottom of both heights, with the farthest point of the pool approximately $1.3 \mathrm{~m}$ from the base of the height. All measurements were taken using an ACE brand measuring tape (7.62 m long).

An imagery task was used to induce fear in the participants and to highlight the potential consequences of falling from the height. In both conditions (dangerous and neutral), participants performed a practice imagery task in either a hallway of the academic building (short height) or on a brick path outdoors (medium height), for which they simply imagined jumping as far as they could across a flat horizontal surface. In the dangerous condition, participants performed an imagery task that involved losing their balance and falling onto the bed of nails. The neutral group imagined losing their balance and landing safely in the empty pool below. Both the practice and experimental imagery tasks were adapted from Clerkin et al (2009).

Finally, trait and state measures of fear were given, which included the Acrophobia Questionnaire anxiety subscale (AQ) (Cohen 1977), Body Sensations Questionnaire (BSQ) (Chambless et al 1984), and a demographic questionnaire. The AQ is a 20-item measure of trait level fear of heights, with each item measured on a scale of 0 (not at all) to 6 (very anxious). The BSQ asked for ratings of bodily sensations experienced during exposure to the height from 0 (none) to 4 (severe).

2.1.3 Procedure. Participants were greeted away from the heights, and were randomly assigned to either the dangerous or neutral condition and to a height location (short or medium). No mention of fear was made and participants did not see the pool of nails until later. Participants then completed the practice imagery task. They were told to keep their eyes closed and to imagine the scene recited to them while standing in a hallway. Briefly, they were asked to imagine jumping as far as they could along a horizontal plane. While still standing in the hall, they were then asked to rate how afraid they were on a $0-100$ scale ( 0 being not at all, and 100 being terrified) so as to get a base level of fear for all of the participants. Finally, they were asked to report, on a $0-100$ scale, how well they imagined performing the practice imagery task (0 being not at all, and 100 being a vivid image).

Upon completing these tasks, the participants were moved to the height location, where their attention was brought to the pool at the bottom of the height. There, the experimenter read them an imagery induction script (adapted from Clerkin et al 2009) while the participants' eyes were closed. For the dangerous condition, participants were told to imagine losing their balance while waving to a friend walking by and falling onto the bed of nails. They were told to form an image of the fall that included multiple sensory experiences and to relate it to previous, real memories of falling. For the neutral condition, participants were told to imagine losing their balance in the same manner as in the dangerous condition, but that they safely landed in the middle of the empty pool (for a complete description of the imagery task read to participants, see Appendix). All participants were told to replay the image in their minds for about $30 \mathrm{~s}$. Following this task, all participants then rated, with their eyes still closed, how afraid they were on the same $0-100$ scale.

Participants were then asked to give height and size estimates. The first estimate of the height was a visual matching task. Participants were told to make the experimenter walk backwards in a straight line away from the participant until the horizontal, ground distance between the participant and the experimenter was equal to the vertical distance from the participants' feet to the ground (the bottom of the pool). Participants were encouraged to look back and forth between the pool and the experimenter as much as they liked. The second estimation task was another visual matching task, 
for which the participants estimated the size of the pool. The experimenter held a tape measure (blank side facing the participants), and the participant told the experimenter to adjust the tape measure until it matched the diameter of the pool. Again, participants were reminded to look back and forth at the pool as much as they liked. This task served as a measure of how large the potentially dangerous area appeared to participants, as well as an indirect measure of height, given previous work has shown perceived size can be an index of perceived height (Stefanucci and Proffitt 2009).

After completing the perceptual tasks, participants were asked to rate the likelihood of injury if they were to fall from the height as depicted in the imagery task ( 0 being no injury, and 100 being death) and how well they visualized falling as described in the imagery task ( 0 being not at all, and 100 being a vivid image of self falling). The participants were then escorted to the laboratory so that they could complete the AQ and BSQ, as well as the demographic questionnaire. The experiment took 15 min to complete.

\subsection{Results and discussion}

2.2.1 Imagery ratings. First, tests were performed to evaluate whether the participants in the two conditions (dangerous and neutral) and the two locations were equally able to complete the imagery tasks both before and during the experimental manipulation. A 2 (condition: danger versus neutral) $\times 2$ (location: short versus medium) $\times 2$ (imagery: practice versus falling) mixed ANOVA with imagery score as the dependent variable revealed no difference between ratings for the practice imagery task (short, $M=67.54$, $\mathrm{SD}=21.1 ;$ medium, $M=70.87, \mathrm{SD}=12.49$ ) and the falling imagery task (short, $M=62.17, \mathrm{SD}=21.7$; medium, $M=70.91, \mathrm{SD}=18.81)$ across condition $\left(F_{1,43}=3.34\right.$, $p=0.08)$, or location $\left(F_{1,43}=1.71, p=0.20\right)$. There was no main effect of imagery $\left(F_{1,43}=0.73, p=0.40\right)$ and no interactions. These data suggest that participants performed the imagery task in a similar manner across conditions, across locations, and across time points in the experiment.

2.2.2 Injury ratings. A 2 (condition: danger versus neutral) $\times 2$ (location: short versus medium height) ANOVA revealed that participants in the dangerous condition rated their likelihood of injury $(M=59.55, \mathrm{SD}=5.61)$ as being significantly higher than those in the neutral condition $(M=13.09, \mathrm{SD}=5.84)\left(F_{1,43}=69.51, \mathrm{MSE}=367.78\right.$, $\left.p<0.0001, \eta_{\mathrm{p}}^{2}=0.62\right)$. Also, participants at the medium height rated their likelihood of injury $(M=43.64, \mathrm{SD}=4.13)$ as higher than participants at the short height $(M=29.0, \mathrm{SD}=3.97)\left(F_{1,43}=7.08, \mathrm{MSE}=367.78, p=0.01, \eta_{\mathrm{p}}^{2}=0.14\right)$. There was no location $\times$ danger interaction $\left(F_{1,43}=0.17, p=0.68\right)$. These data suggest that the consequences for falling were perceived to be higher in the dangerous condition than the neutral condition and at the medium height in comparison to the short height; therefore, the manipulation of consequences at the heights was successful and the heights themselves inherently differed in threat, but were not very threatening overall given the low means for the injury ratings in the neutral condition.

2.2.3 Fear ratings. A mixed ANOVA revealed that the fear ratings (SUDS) were significantly higher following the experimental imagery task $(M=39.28, \mathrm{SE}=4.29)$ as compared to the practice imagery task $(M=7.07, \mathrm{SE}=1.67)\left(F_{1,44}=60.87\right.$, $\left.\mathrm{MSE}=407.537, p<0.0001, \eta_{\mathrm{p}}^{2}=0.58\right)$ for both locations. As expected, the average fear ratings did differ by condition $\left(F_{1,44}=5.79, \mathrm{MSE}=604.54, p=0.02, \eta_{\mathrm{p}}^{2}=0.12\right)$ such that participants who were in the danger condition gave significantly higher fear ratings than participants in the neutral condition. There were no differences in ratings for each location $\left(F_{1,44}=0.07, p=0.79\right)$, suggesting that changes in fear were equivalent and there was no condition $\times$ location interaction $\left(F_{1,44}=0.28, p=0.59\right)$. 
Finally, there were no differences among the groups in trait-level fear of heights or in the bodily sensations experienced at the height. A multivariate ANOVA testing for differences across danger and location for the AQ was not significant (danger: $F_{1,44}=0.00, p=0.99$; location: $F_{1,24}=0.13, p=0.72$ ), nor was a test for differences in the BSQ (danger: $F_{1,44}=0.35, p=0.56$; location: $F_{1,44}=1.50, p=0.23$ ). There were also no interactions between any of the factors on either measure. These data are important because they reveal no preexisting height fear differences among the participants in each group.

2.2.4 Perceptual estimates. Given participants viewed different heights at each location, we converted both the height and size estimates to ratios in order to compare across location. Ratios were calculated by dividing the estimated height by the actual height and the estimated size by the actual size. Thus, numbers greater than 1 represent overestimations and less than 1 represent underestimations. A 2 (condition: danger versus neutral) $\times 2$ (location: short versus medium) ANOVA with ratio-scaled visually matched estimates of the height as the dependent variable revealed that participants in the dangerous condition overestimated the height more $(M=1.84, \mathrm{SE}=0.07)$ than participants in the neutral condition $(M=1.34, \mathrm{SE}=0.07)\left(F_{1,43}=25.58, \mathrm{MSE}=0.12\right.$, $p<0.0001, \eta_{\mathrm{p}}^{2}=0.37$ - see figure 1a). Not surprisingly, participants at the medium height overestimated the height more $(M=1.70, \mathrm{SE}=0.07)$ than participants at the short height $(M=1.48, \mathrm{SE}=0.07)\left(F_{1,43}=4.66, \mathrm{MSE}=0.12, p=0.04, \eta_{\mathrm{p}}^{2}=0.10\right.$ - see figure 1a). Finally, there was a condition $\times$ location interaction, such that participants who were at the medium height showed a larger increase in visually matched estimates when in the dangerous condition as compared to participants in the short, dangerous condition $\left(F_{1,43}=7.30, \mathrm{MSE}=0.12, p=0.01, \eta_{\mathrm{p}}^{2}=0.15\right.$ - see figure 1a). Another 2 (condition: danger versus neutral) $\times 2$ (location: medium versus short) ANOVA with the pool size estimates as the dependent variable, showed that participants in the dangerous condition saw the pool as larger $(M=1.04, \mathrm{SE}=0.02)$ than those in the neutral condition $(M=0.94, \mathrm{SE}=0.02) \quad\left(F_{1,43}=9.78, \mathrm{MSE}=0.01, p=0.003\right.$, $\eta_{\mathrm{p}}^{2}=0.19$ - see figure 1b). Also, participants at the medium height overestimated the size of the pool $(M=1.09, \mathrm{SE}=0.03)$ in comparison to participants at the short height $(M=0.89, \quad \mathrm{SE}=0.02) \quad\left(F_{1,43}=36.30, \quad \mathrm{MSE}=0.01, \quad p<0.0001, \quad \eta_{\mathrm{p}}^{2}=0.46\right)$. There was no condition $\times$ location interaction for size estimates $\left(F_{1,43}=0.07, p=0.80\right)$.
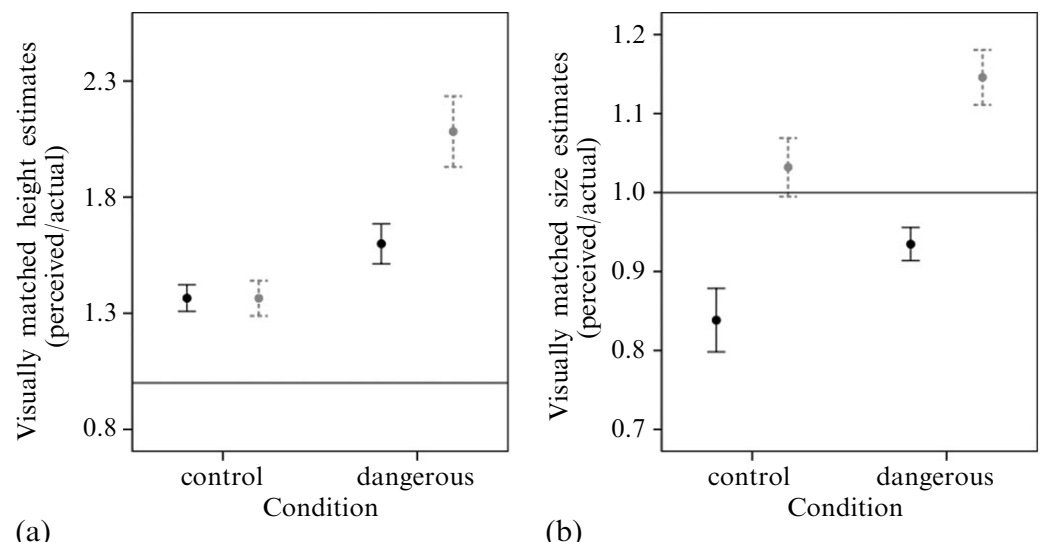

Figure 1. (a) Visually matched estimates (ratio scaled by dividing perceived height by actual height) of the short and medium heights for each condition in experiment 1. (b) Visually matched size estimates (ratio scaled by dividing perceived size by actual size) of the pool at both the short and medium heights for each condition in experiment 1 . For both figures, the bars represent 1 standard error and the horizontal lines denote the actual height of the balcony and size of the pool. The black bars represent the ratios for the short height and the gray bars for the medium height. 
It should be noted that, in general, the size of the pool was underestimated for the short location. This finding is not surprising, given that the participants were very close to the pool, and previous research has found that size is generally underestimated, especially when the to-be-judged object is emotionally negative (van Ulzen et al 2008).

\section{Experiment 2}

This experiment extended the findings from the previous experiment to the assessment of horizontal ground distances. Participants in the dangerous condition were asked to imagine jumping across the pool of nails and the participants in the neutral condition were asked to imagine jumping across an upside-down pool. This experiment allowed us to determine whether the imagined costs associated with an action would also affect the estimation of horizontal ground distances that are not inherently dangerous.

\subsection{Method}

3.1.1 Participants. Twenty-six (nineteen female, seven male) undergraduate students (mean age $=18.77$ years, $\mathrm{SD}=0.99$ years) participated in the study to fulfill a course requirement.

3.1.2 Stimuli and apparatus. The same inflatable pool was used as in experiment 1. To equate pool height across conditions, the pool was set upside-down so that the flat underside of the pool faced the participants in the neutral condition, which maintained the same height as the maximum height of the nails in the dangerous condition. Participants stood in a $20.57 \mathrm{~m}$ hallway to make their estimates of horizontal ground distance. A small, orange construction cone marked the distance $(1.83 \mathrm{~m})$ to be judged on the opposite side of the pool from participants. All other apparati were the same as in experiment 1.

3.1.3 Procedure. Participants were greeted away from the experimental site, randomly assigned to condition, and told that they would be jumping over a small inflatable pool. As a cover story, they were told that previous research had shown that imagining an action prior to performing it improves performance and that this study would focus on that relationship between imagery and jumping. Then participants were given the instructions for the practice imagery task, as in the previous experiment. The participants performed the practice imagery task away from the experimental site. Once completed, the participants were asked the same questions from experiment 1 about how well they imagined jumping, and how afraid they felt at that moment $(0-100$ scales $)$.

Participants were then escorted to the experimental hallway. The pool was located $0.45 \mathrm{~m}$ from the front of the participants' feet, and an orange construction cone was located just beyond the pool at $1.83 \mathrm{~m}$ from the front of participants' feet. The participants in the dangerous condition completed the experimental imagery task, which involved imagining jumping over a pool of nails, losing their balance, and falling helplessly onto the nails. Control participants were asked to perform a similar task, but they imagined successfully jumping over the flat pool with no mention of loss of balance. After participants in both conditions had run the image through their minds a few times with their eyes closed, they were asked to relay their level of fear on the $0-100$ scale ( 0 being none, 100 being terrified), and their ability to imagine the scenario on the $0-100$ scale.

The participants were then told to open their eyes and perform three perceptual tasks. They were first asked to estimate the size of the pool by performing the sizematching task described in experiment 1 . They then performed a similar matching task, but replicated the extent of the horizontal distance between their feet and the closest edge of the pool $(0.45 \mathrm{~m})$ with the tape measure. The last matching task involved asking the researcher to walk backwards perpendicularly from the horizontal extent between the 
participant and the orange cone until the ground distance between the researcher and the participant was the same as between the participants' feet and the orange cone on the other side of the pool. Participants then reported how well they had imagined performing the jump as described by the experimenter, and how injured they would be if they fell as described in the dangerous and control imagery tasks, respectively. Finally, participants were informed that they would not have to jump over the pool as they had previously been told. They were then asked to fill out a demographic questionnaire and the BSQ. The experiment took 15 min to complete.

\subsection{Results and discussion}

3.2.1 Imagery ratings. A one-way ANOVA revealed no difference in participants' reported abilities to imagine jumping during the practice imagery task between the dangerous condition $(M=68.85, \mathrm{SD}=22.09)$ and the neutral condition $(M=68.15, \mathrm{SD}=21.69)$ $\left(F_{1,24}=0.007, p=0.94\right)$. There was also no difference in participants' reported ability to imagine jumping over the pool between the dangerous condition $(M=78.08$, $\mathrm{SD}=16.90)$ and the neutral condition $(M=67.92, \mathrm{SD}=24.01)\left(F_{1,24}=1.55, p=0.23\right)$. A mixed ANOVA with imagery score as the dependent variable and condition as the between-participants factor revealed no difference between ratings for the practice imagery task and the jumping imagery task across condition $\left(F_{1,24}=0.63, p=0.43\right)$. There was no main effect of imagery $\left(F_{1,24}=0.86, p=0.36\right)$ and no condition $\times$ imagery interaction $\left(F_{1,24}=0.95, p=0.34\right)$. These data suggest that participants performed the imagery task in a similar manner across conditions and across time points in the experiment.

3.2.2 Manipulation of danger. A one-way ANOVA revealed that participants in the dangerous condition rated their likelihood of being injured from falling as significantly higher $(M=70.15, \mathrm{SD}=24.32)$ than that in the neutral condition $(M=6.46, \mathrm{SD}=8.09)$ $\left(F_{1,24}=116.16, \mathrm{MSE}=328.46, p<0.0001, \eta_{\mathrm{p}}^{2}=0.77\right)$. These data suggest that the consequences for falling when jumping were perceived to be higher in the dangerous condition than the neutral condition; therefore, the manipulation was successful.

3.2.3 Fear ratings and measures. A mixed ANOVA was run comparing SUDS ratings before and after the jumping imagery task for both conditions. There was a main effect of condition; participants in the dangerous condition reported feeling more fear than participants in the neutral condition $\left(F_{1,24}=20.54, \mathrm{MSE}=323.64, p<0.0001\right.$, $\left.\eta_{\mathrm{p}}^{2}=0.46\right)$. There was a main effect of fear rating, such that there was a significant change in fear from the practice imagery task to the jumping imagery task regardless of condition $\left(F_{1,24}=64.39, \mathrm{MSE}=310.73, p<0.0001, \eta_{\mathrm{p}}^{2}=0.73\right)$. There was also a fear $\times$ condition interaction $\left(F_{1,24}=12.76, \mathrm{MSE}=310.73, p=0.002, \eta_{\mathrm{p}}^{2}=0.35\right)$, such that participants in the dangerous condition showed a larger increase in fear ratings between the practice and jumping imagery tasks than participants in the neutral condition. Finally, a one-way ANOVA testing for differences across conditions for the BSQ failed to reach significance $\left(F_{1,25}=3.58, p=0.07\right)$.

3.2.4 Perceptual estimates. A one-way ANOVA comparing visually matched estimates of the distance estimates across conditions revealed that participants in the dangerous condition perceived the distance to the pool from their feet to be farther $(M=0.48 \mathrm{~m}$, $\mathrm{SD}=0.06 \mathrm{~m})$ than participants in the neutral condition $(M=0.42 \mathrm{~m}, \mathrm{SD}=0.05 \mathrm{~m})$ $\left(F_{1,24}=8.65, \mathrm{MSE}=0.003, \quad p=0.007, \eta_{\mathrm{p}}^{2}=0.27\right.$ - see figure $\left.2 \mathrm{a}\right)$. Similarly, participants in the dangerous condition estimated the distance to the cone (across the pool of nails) to be farther $(M=2.04 \mathrm{~m}, \mathrm{SD}=0.26 \mathrm{~m})$ than in the neutral condition $(M=1.80 \mathrm{~m}, \mathrm{SD}=0.19 \mathrm{~m})\left(F_{1,24}=7.11, \mathrm{MSE}=0.05, p=0.01, \eta_{\mathrm{p}}^{2}=0.23\right.$ - see figure $\left.2 \mathrm{~b}\right)$. However, the size estimates of the pool did not differ by condition $\left(F_{1,24}=0.00, p=0.99\right)$. 


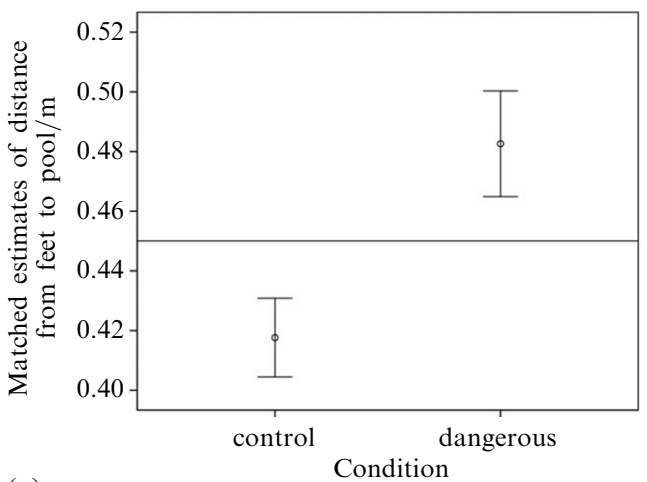

(a)

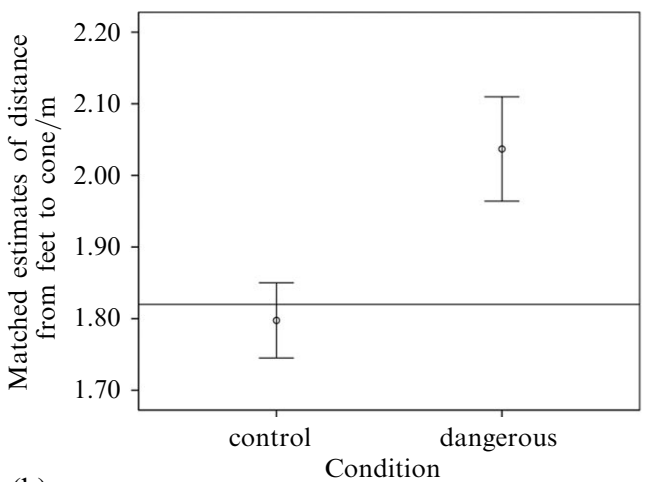

(b)

Figure 2. (a) Visually matched estimates of the distance from the feet to the pool and (b) the feet to the target cone across the pool in experiment 2. For both figures, the bars represent 1 standard error and the lines denote the actual distances.

These data suggest that participants in the dangerous condition saw distances to the pool and across the pool as farther when they imagined jumping across the pool, but did not overestimate the size of the pool itself more than the participants in the neutral condition.

There are a few reasons why the size overestimation of the pool may not have occurred in this experiment. First, participants were closer to the pool in this experiment than in the previous experiment. Therefore, the size of the pool may have been more apparent to them given their proximity to it. As in experiment 1 (when participants at the short height were also closer to the pool), a general underestimation of size was observed. On average, both groups estimated the size of the pool to be almost exactly the same: $1.01 \mathrm{~m}$ in diameter. This represents a general underestimation of size given that the true size of the pool was $1.17 \mathrm{~m}$. Again, participants may have also underestimated the size of the pool because it was a negatively valenced target object, which has been shown to result in the underestimation of size in past research (van Ulzen et al 2008). Another possibility is that the participants were asked to focus on jumping over the pool to the orange cone, so fear may only have altered the perceived distance needed to clear the nails and hit the target, rather than taking into account the size of the pool. In other words, participants may not have needed to see the size of the pool as larger if they already overestimated the distance needed to reach the target. Fear may have only changed the perception needed to meet the task demand (jumping over the pool to the cone).

\section{General discussion}

These experiments show that directly manipulating the consequences of falling can influence estimates of non-threatening vertical and horizontal distances. These findings are the first to suggest that direct manipulations of the consequences of action can influence multiple parameters of spatial layout, not just heights. Previous research has only investigated the influence of fear on estimates of tall heights ( $\sim 6 \mathrm{~m}$ or higher) and with populations of individuals who are afraid of heights. These studies also found that manipulations of danger can affect size estimation of real objects, given participants estimated a dangerous object (the bed of nails) as larger when they imagined falling into it. These findings support prior work showing size estimates of images are affected by fear (see van Ulzen et al 2008).

A question remains. Did people who imagined falling into a bed of nails actually see the extent as different, or did they just report that it was different? Given that participants in these experiments were told to imagine falling or jumping and their attention was directed to a threatening environment in the dangerous condition, 
they could have intuited our hypotheses and biased their responses accordingly. We cannot be sure that participants were not doing this; however, we used multiple dependent measures to try to control for this potential bias. For example, participants may have believed that we wanted them to say that the height was higher, but it is less clear that they would know that saying the pool was larger would also indicate that the height looked taller (the size-distance invariance hypothesis-Epstein 1973). Admittedly, they may have felt compelled to bias both responses, but that would have been most likely in the dangerous conditions. Also, participants in experiment 2 did not overestimate the size of the pool, possibly because they were closer to the pool. However, if they had biased their distance responses because they believed we expected them to say that the distance was farther (especially in the dangerous condition), then it is likely that they would have altered their estimates of the size of the pool as well. For the experiments reported in this paper, we believe participants' visual systems were employing an adaptive perceptual strategy. A visual system that recognizes the costs associated with acting in the environment and biases the perception of that environment in order to reduce dangerous actions would be extremely adaptive and economical (Proffitt 2006; Stefanucci et al 2011).

There is also the possibility that, because our measure involved matching to a sample, participants had to look away from the distance being judged to set the experimenter at the appropriate place, thereby rendering the judgment one from memory rather than from perception. Though the time between the participants' visual perception of the stimuli and their verbal reports was brief, it is possible that the costs associated with acting influence the memory of the perception rather than the perception itself, especially if the memory of the perception is less stable than the direct perception. Recent research suggests that effects in which non-visual factors are claimed to influence perception may be due to temporal aspects of the procedure that could evoke changes in memory rather than changes in perception (Bridgeman et al 2011). In the future, it would be helpful to run a condition in which participants give verbal or haptic responses while viewing the distance itself to control for changes in memory.

Also, estimates of the costs associated with acting (or falling) in the environment were related to actual ratings of fear, though may not have been the direct result of fear. In both experiments, participants rated their likelihood of injury to be very high in the dangerous condition, and also reported an increase in fear after imagining falling, even though participants in the height conditions likely knew that they would not actually be jumping or falling from the height. Therefore, they recognized the costs associated with falling. Participants in experiment 2 may have believed they would have to jump over the threatening pool, given their fear ratings did increase. Therefore, when action became a real possibility, fear did increase. Another alternative is that the height was a high-anxiety situation to begin with and the fear rating (a single-item measure) may not have been sensitive enough to detect differences when the costs of falling were increased in an already threatening environment (see Tryon 1977 for a similar finding). We are confident from the results with the injury ratings that participants perceived a change in the costs associated with action in the environment across the dangerous and neutral conditions. Whether a change in fear is also needed to alter perceived distance and size remains an open question. However, to separate out perceived fitness costs from fear responses is difficult, perhaps because the adaptive function of fear is to prepare the organism to deal with immediate threats like looming predators and falling from dangerous heights - situations in which the cost of action is enormous.

This work contributes to a growing body of research examining the influence of action costs on perception or action-specific effects on perception (see Proffitt 2006 and Witt 2011, respectively). However, it also adds important new findings to that research. 
We showed that altering the costs associated with acting on non-dangerous extents (heights and horizontal distances) and asking participants to imagine a dangerous outcome for those actions significantly increased participants' estimates of the environment (both extent and size). Neither the economy of action approach (Proffitt 2006) nor the actionspecific effects on perception approach (Witt 2011) have addressed the influence of manipulations of dangerous action on perception. The findings of experiment 2 , in which increased costs associated with acting on a horizontal ground distance influenced estimates of that distance is a definitive departure from the previous work on height perception, but does provide further support for Proffitt's and Witt's claims. When an observer imagined a dangerous outcome for an otherwise non-dangerous action (eg jumping across an inflatable pool), estimates of horizontal ground distance to the dangerous area and across it were enlarged.

Previous research showed that imagining a fall could lead participants who were high in height fear to overestimate heights more (Clerkin et al 2009). In this paper, we found that an average, non-phobic population will also overestimate heights more when the costs associated with acting in an environment are made large and participants are asked to imagine a dangerous outcome for their actions. Our findings suggest that increased threat at a height or ground distance (that is visual and not just imagined) may influence participants' overestimation of those distances.

Acknowledgments. This research was supported in part by NIH RO1MH075781-01 A2 grant, for which the first author served as a consultant. The authors would like to thank Dorian Rosen for her help in collecting and analyzing the data.

\section{References}

Bridgeman B, Cooper A, Sterling C, Bacon M, 2011 "Temporal aspect of motor performance's effect on perception" Journal of Vision 11956

Chambless D L, Caputo G C, Bright P, Gallagher R, 1984 "Assessment of fear in agoraphobics: The Body Sensations Questionnaire and the Agoraphobic Cognitions Questionnaire" Journal of Consulting and Clinical Psychology $521090-1097$

Clerkin E M, Cody M W, Stefanucci J K, Proffitt D R, Teachman B A, 2009 "Imagery and fear influence height perception" Journal of Anxiety Disorders 23381 - 386

Cohen D, 1977 "Comparison of self-report and behavioral procedures for assessing acrophobia" Behavior Therapy 8 17-23

Epstein W, 1973 "The process of 'taking-into-account' in visual perception" Perception 2 267-285

Jackson R, Cormack L, 2007 "Evolved navigation theory and the descent illusion" Perception \& Psychophysics $\mathbf{6 9} 353-362$

Proffitt D R, 2006 "Embodied perception and the economy of action" Perspectives on Psychological Science $1110-122$

Pylyshyn Z W, 2003 "Explaining mental imagery: Are there really pictures in the brain?" Trends in Cognitive Sciences $7113-118$

Stefanucci J K, Gagnon K T, Lessard D, 2011 "Follow your heart: Emotion adaptively influences perception" Social Psychology and Personality Compass 5/6 296-308

Stefanucci J K, Proffitt D R, 2009 "The roles of altitude and fear in the perception of height" Journal of Experimental Psychology: Human Perception and Performance 35 424-438

Teachman B A, Stefanucci J K, Clerkin E M, Cody M W, Proffitt D R, 2008 "A new mode of fear expression: perceptual bias in height fear" Emotion 8 296-301

Tryon W W, 1977 "Psychophysical scaling and hierarchy construction" Journal of Behavior Therapy and Experimental Psychiatry $853-56$

Ulzen N R van, Semin G R, Oudejans R R D, Beek P J, 2008 "Affective stimulus properties influence size perception and the Ebbinghaus illusion" Psychological Research 72 304-310

Witt J K, 2011 "Action's effect on perception" Current Directions in Psychological Science 20 $201-206$ 


\section{Appendix}

\section{Dangerous condition imagery instructions}

I want you to imagine this situation not as if you were an actor in a play, but as if you were really there, looking out at it through your own eyes. Please close your eyes and imagine the following occurring, please keep your eyes closed until further instructed. Imagine you are standing at the edge of the loading dock looking down at the pool and the bed of nails contained within it. While looking down into the pool you hear your name shouted by a friend who is walking by the loading dock on her way to class. Instinctively you look up in response and while shifting your gaze you unconsciously lift your right foot to step in her direction when you realize you are still on the edge of the loading dock. You immediately look down at your foot hanging over the edge and a rush of adrenaline shoots through your body as you arch your back in an effort to shift your body weight. Still you've lost your balance too much to be able to regain your footing and you spin rapidly around as you fall backwards reaching your hand out to the researchers but your fingers narrowly slip through their grasp and you look at their horrified facial expressions as you helplessly fall backwards. Even expecting an immense level of pain does not prepare you for the pounding strike that occurs as you hit the bed of nails and pavement, Now I want you to hold that image in your mind and replay it over and over for $30 \mathrm{~s}$.

\section{Neutral condition imagery instructions}

I want you to imagine this situation not as if you were an actor in a play, but as if you were really there, looking out at it through your own eyes. Please close your eyes and imagine the following occurring, please keep your eyes closed until further instructed. Imagine you are standing at the edge of the loading dock looking down at the empty pool below. While looking down into the pool you hear your name shouted by a friend who is walking by the loading dock on her way to class. Instinctively you look up in response and while shifting your gaze you unconsciously lift your right foot to step in her direction when you realize you are still on the edge of the loading dock. You immediately look down at your foot hanging over the edge of the wall. Instinctively you shift your body weight to regain your footing and you jump and land flatly and safely in the middle of the empty pool. Now I want you to hold that image in your mind and replay it over and over for $30 \mathrm{~s}$. 


\section{PERCEPTION}

VOLUME 412012

www.perceptionweb.com

Conditions of use. This article may be downloaded from the Perception website for personal research by members of subscribing organisations. Authors are entitled to distribute their own article (in printed form or by e-mail) to up to 50 people. This PDF may not be placed on any website (or other online distribution system) without permission of the publisher. 